\title{
BENEFÍCIOS DA CAFEÍNA SOBRE OS NÍVEIS SÉRICOS DE COLESTEROL E TRIGLICERÍDEOS
}

ARAÚJO, Tatiana O. P.; GOES, Liz Evelyn de Jesus de; ROCHA, Aline G.; COSTA, Marciano Pires da;

SANTOS, Luiz Henrique S. Biomédicos formados pelo Centro Universitário de Araraquara - Uniara. BERTATO, Aline C. Técnica do Laboratório de Biologia (Interlab) do Centro Universitário de Araraquara - Uniara.

GILENO, Miriane da Costa. Mestre e Doutora em Análises Clínicas (FCF-UNESP), professora de Hematologia Clínica e Bioquímica e Enzimologia Clínica - Uniara e professora de Hematologia Clínica nos Cursos de Especialização em Análises Clínicas e Hematologia e Hemoterapia - Barão de Mauá. Curso de

Biomedicina. Laboratório de Hematologia Clínica - Uniara. Tel: (16) 3331-5985. E-mail:

mcostagileno@yahoo.com.br.

\section{RESUMO}

A cafeína (CF) é o ingrediente ativo do café e também de outros alimentos e medicamentos muito consumidos. Este estudo teve como objetivo avaliar o efeito da $\mathrm{CF}$, em diferentes concentrações, sobre os valores séricos de colesterol total (CT) e frações, triglicerídeos (TG) e lipoproteínas HDL colesterole VLDL colesterol em ratos. Foram analisadas 60 amostras de soro de ratos Wistar machos, submetidos à ingestão de solução aquosa de CF durante quatro dias, sendo sacrificados no quinto dia. As análises de CT e frações foram realizadas por equipamento automatizado (Technicon RA-XT) e os valores de VLDL colesterol, calculados pela equação de Friedewald. Os ratos submetidos ao tratamento com CF foram divididos em três grupos: a) grupo 1: 0,3 mg/ $\mathrm{mL} / 100 \mathrm{~g}$ peso; b) grupo 2: $6 \mathrm{mg} / \mathrm{mL} / 100 \mathrm{~g}$ peso; e c) grupo $3: 10 \mathrm{mg} / \mathrm{mL} / 100 \mathrm{~g}$ peso. Ao grupo controle não foi administrada CF. Foi observada uma diminuição altamente significativa $(\mathrm{p}<0,01)$ nos valores de $\mathrm{CT}$ dos ratos pertencentes aos grupos 2 e 3 em relação aos do grupo controle; detectou-se uma diminuição altamente significativa $(\mathrm{p}<0,01)$ nos valores de TG e VLDL colesterol dos ratos pertencentes ao grupo 3 quando comparados aos do grupo controle; verificou-se também uma diminuição altamente significativa $(\mathrm{p}<0,01)$ nos valores de HDL colesterol dos ratos pertencentes ao grupo 2 quando comparados aos do grupo controle. Foi demonstrado também um efeito dose-dependente nos grupos tratados com CF. Estudos epidemiológicos demonstram que o consumo de café fervido e não filtrado eleva o risco de doenças cardiovasculares, devido à presença de dois diterpenos, que promovem o aumento da concentração plasmática de colesterol em seres humanos. Entretanto, outros estudos mostram que o consumo de substâncias ricas em CF leva à diminuição de CT. Nosso trabalho demonstrou que a cafeína pura levou à diminuição de CT, TG e VLDL colesterol, sugerindo um efeito benéfico da CF no sistema cardiovascular, principalmente quando associada aos antioxidantes presentes no café. No entanto, em razão da diminuição nos níveis de HDL colesterol, serão necessários estudos complementares para esclarecer seu exato papel no organismo e na prevenção da placa de ateroma.

Palavras-chave: Cafeína; Lipídeos e lipoproteínas.

\section{Abstract}

Caffeine is the active ingredient of coffee and also of other foods and most consumed medicines. The purpose of this study was to evaluate the effect of caffeine at different concentrations on the plasmatic values of total cholesterol (CT), triglycerides (TG), and lipoproteins HDL and VLDL in mice. We have analysed 60 samples of serum of male mice Wistar, which had undergone the ingestion of aqueous solution of caffeine during four days, being sacrificed in the fifth day. The analyses ofCT, TG and HDL were carried out by automated equipment (Technicon RA-XT) and the values of VLDL cholesterol were calculated with Friedewald equation. The mice 
that had undergone treatment with caffeine were divided in 3 groups: a) group 1: 0,3 mg/mL/100g weigh; b) 6 group $2: \mathrm{mg} / \mathrm{mL} / 100 \mathrm{~g}$ weigh and c) 10 group 3 : $\mathrm{mg} / \mathrm{mL} / 100 \mathrm{~g}$ weigh. The control group was not given any caffeine. It was observed a highly significant reduction $(p<0,01)$ in the values of CT of the mice belonging to groups 2 and 3 regarding the control groups; a highly significant reduction was observed ( $p$ $<0,01)$ in the values of TG and VLDL of the mice belonging to group 3 when compared to the control group; there was also a highly significant reduction ( $\mathrm{p}$ $<0,01$ ) in the values of HDL of the mice belonging to group 2 when compared to the control group. It also was demonstrated a dose - dependent effect in the groups treated with caffeine. Epidemiological studies have demonstrated that the consumption of hot and not filtered coffee lifts up the risk of cardiovascular diseases, due to the presence of two diterpenes, which promote the increase of the plasmatic concentration of cholesterol in human beings. However, other studies have shown that the consumption of rich in caffeine substances, results in the reduction of total cholesterol. Our work has demonstrated that pure caffeine resulted in the reduction of total cholesterol, triglycerides and VLDL, suggesting a beneficial effect of the caffeine in the cardiovascular system principally when it is associated to antioxidants present in coffee. However due to the decrease in the levels of HDL cholesterol, further studies are needed to clarify its exact role in the body and in the prevention of atherosclerotic plaque.

KEYwORD: Caffeine; Lipids and lipoproteins.

\section{INTRODUÇão}

A cafeína é uma das substâncias mais consumidas no planeta. É uma droga poderosa, porém inofensiva, saborosa, capaz de manter as pessoas despertas e produtivas por mais de três horas, sendo utilizada por isso como droga utilitária. Porém, pode perder o seu efeito ou causar dor de cabeça quando seu consumo ultrapassa os 200 miligramas diários, não importa qual seja a fonte. Adose letal de cafeína para o ser humano é de cerca de 10 gramas, sendo que uma xícara de café contém cerca de $80 \mathrm{mg}$ de cafeína (JAMES, 1997).

São múltiplas as fontes de cafeína, e isso faz com que seja difícil quantificar seu consumo, sendo este maior que o do álcool e nicotina juntos e com maior prevalência entre pacientes psiquiátricos e presidiários (STRAIN; GRIFFITHS, 2000).

A cafeína $\left(\mathrm{C}_{8} \mathrm{H}_{10} \mathrm{~N}_{4} \mathrm{O}_{2}\right)$ ou 1,3,7-trimetilxantina é classificada como um alcaloide pertencente ao grupo de compostos químicos denominados metil-xantinas. As xantinas são substâncias capazes de estimular o sistema nervoso, produzindo um estado de alerta de curta duração. Entre o grupo das xantinas a cafeína é a que mais atua sobre o sistema nervoso central. Atua ainda sobre o metabolismo basal e aumenta a produção de suco gástrico (BUCHALLA, 2008).

A absorção da cafeína no organismo é muito rápida, assim como a sua distribuição, passando rapidamente para o sistema nervoso central. Existe a chamada "sensibilidade à cafeína", a qual se refere à quantidade necessária dessa substância para produzir os efeitos secundários negativos, tais como perda de sono e aumento da frequência cardíaca.

A ação da cafeína no corpo depende da forma de preparo do produto. Por exemplo, alguns estudos demonstram que o consumo de café fervido e não filtrado pode levar ao aumento do risco de doenças coronarianas, devido à presença de dois diterpenos, o cafestol e o kahweol, que promovem o aumento da concentração plasmática de colesterol (RANHEIM; HALVORSEN, 2005). Depende também da quantidade utilizada e das condições do organismo que a consome, podendo o efeito variar de indivíduo para indivíduo.

Contradizendo esses estudos, outros demonstram que o consumo da cafeína está relacionado à sua capacidade de estimular a lipólise (quebra das moléculas de gordura no organismo), através do aumento da liberação de lipase, o que, teoricamente, favoreceria o emagrecimento. A mobilização dos depósitos de gordura pode ser útil para atletas em treinamento intenso, fazendo com que o organismo utilize a gordura como fonte de energia no lugar do glicogênio muscular; com isso, o corpo fica mais 
resistente à fadiga (CAMARGO, 2001).

Perfil lipídico é o conjunto de análises laboratoriais realizado para quantificar os níveis séricos de colesterol total, triglicerídeos e estimar os níveis de lipoproteínas (VLDL, HDL e LDL) juntos.

A análise do perfil lipídico é de extrema importância, uma vez que alguns desses elementos em altas concentrações no organismo podem desencadear problemas cardíacos, circulatórios, entre outros.

Os lipídeos são um grupo heterogêneo de moléculas - mais relacionadas por suas propriedades físicas do que químicas. Têm um importante papel em nosso organismo, participando na constituição de hormônios ou de seus precursores; auxiliam na digestão; servem de armazenamento e fonte de energia metabólica; entre outros papéis.

O colesterol, sintetizado principalmente pelo fígado, é encontrado em todas as células do corpo, mas em maior proporção no cérebro e nos tecidos nervosos; está presente em gorduras de origem animal, assim não sendo encontrado em gordura vegetal.

Os triglicérides são um tipo de gordura que constitui $90 \%$ do armazenamento energético do organismo. Depositam-se no tecido adiposo e muscular e são provenientes da dieta ou produzidos pelas células do fígado (CURI et al., 2002).

Os níveis de lipídeos são relevantes, pois elevados níveis de colesterol total e LDL-colesterol estão intimamente relacionados com riscos de doenças coronarianas isquêmicas. Os níveis de triglicerídeos são de igual importância, pois, se estiverem elevados no soro, são associados a condições patogênicas que aceleram a aterosclerose.

O termo lipoproteína é empregado não para um composto químico definido, mas sim para uma família de partículas, cuja finalidade é transportar lipídeos, principalmente triglicérides e colesterol, entre órgãos e tecidos (CURI et al., 2002). As lipoproteínas possuem um núcleo apolar contendo triglicerídeo e colesterol esterificado, e uma membrana polar contendo colesterol livre, apolipoproteínas e fosfolipídeos. Essas lipoproteínas foram classificadas segundo sua densidade.

Em trabalho anterior foi demonstrado que a cafeína, o ingrediente ativo do café, e também encontrada em outros alimentos e bebidas muito consumidas, como chocolate, cháe alguns analgésicos, pode ter diversos efeitos sobre o perfil lipídico. Devido ao alto consumo de café e desses outros alimentos, pode-se dizer que cerca de $80 \%$ da população geral faz uso desta substância diariamente, embora seja muito difícil quantificar seu consumo (STRAIN; GRIFFITHS, 2000), que pode ter ação sobre o perfil lipídico, levando à diminuição dos níveis séricos de alguns elementos.

Muitos trabalhos (INTERNATIONAL FOOD INFORMATION COUNCIL FOUNDATION-IFIC, 1999) têm avaliado o efeito da cafeína sobre os valores de colesterole triglicerídeos, devido às evidências de que o aumento dos níveis de triglicerídeos é um fator de risco independente para aterosclerose e de que a determinação do colesterol total e HDL colesterol, em amostras de sangue, é útel na investigação de dislipidemias e faz parte da avaliação do risco de doenças coronarianas isquêmicas. No entanto, esses trabalhos se têm mostrado contraditórios, justificando a importância do presente estudo.

\section{ObJetivo}

Este estudo teve como objetivo avaliar o efeito da cafeína, em diferentes concentrações, sobre os valores séricos de colesterol total e frações e triglicerídeos em ratos.

\section{Material e métodos}

Foram analisadas 60 amostras de soro de ratos Wistar machos, submetidos à ingestão, por meio de cânula, de solução aquosa de cafeína anidra durante quatro dias, sendo sacrificados por deslocamento de cervical no quinto dia (aprovado junto ao Comitê de Ética do Centro Universitário de Araraquara- Uniara). As análises de colesterol total (CT), triglicerídeos (TG) e do colesterol ligado à lipoproteína de alta densidade (HDL colesterol) foram realizadas por equipamento bioquímico automatizado (Technicon RA-XT), e os valores de colesterol ligado à lipoproteína de muito baixa densidade (VLDL colesterol), estimados pela Equação de Friedewald. Como os valores de 
colesterol ligado à lipoproteína de baixa densidade (LDL colesterol) variaram muito entre os ratos do mesmo grupo, não foi possível demonstrar os resultados neste trabalho.

Equação de Friedewald:

(1) VLDL colesterol $=\frac{\text { Triglicerídeos }}{5}$

(2) LDL colesterol $=$ Colesterol total $-($ HDL colesterol + VLDL colesterol)

Obs.: Esta equação só poderá ser aplicada para valores de triglicerídeos $<400 \mathrm{mg} / \mathrm{dL}$.

Os ratos submetidos ao tratamento com cafeína foram divididos em três grupos: a) grupo 1: 0,3 mg/ $\mathrm{mL} / 100 \mathrm{~g}$ peso; b) grupo $2: 6 \mathrm{mg} / \mathrm{mL} / 100 \mathrm{~g}$ peso e c) grupo 3: $10 \mathrm{mg} / \mathrm{mL} / 100 \mathrm{~g}$ peso. Ao grupo controle não foi administrada cafeína.

A análise estatística foi realizada comparando os grupos em que foram administradas diferentes concentrações de cafeína e também frente ao grupo controle. Os resultados foram calculados como média +/- desvio padrão e as análises estatísticas, realizadas pelo teste $-\mathrm{F}$ (duas amostras para variâncias), seguido do teste $-\mathrm{t}$, que estabeleceu o nível de significância de $\mathrm{p}<0,05$.

\section{Resultados}

Foi observada uma diminuição altamente significativa $(\mathrm{p}<0,01)$ nos valores de colesterol total (Figura 1) dos ratos pertencentes aos grupos 2 e 3 em relação aos do grupo controle e aos do grupo 1 (0,3 mg/mL cafeína).

Verificou-se uma diminuição altamente significativa $(\mathrm{p}<0,01)$ nos valores de triglicérides (Figura 2) dos ratos pertencentes ao grupo 3 quando comparados aos do grupo controle e aos dos grupos com doses mais baixas de cafeína.

Com relação ao colesterol ligado à lipoproteína de alta densidade (HDL colesterol) (Figura 3), observouse uma diminuição altamente significativa $(\mathrm{p}<0,01)$ nos valores deste nos ratos pertencentes ao grupo 2 quando comparados aos do grupo controle, e uma diferença significativa $(\mathrm{p}<0,01)$ quando se comparou o grupo 3 (10mg cafeína/mL) com o grupo 1 ( $0,3 \mathrm{mg}$ cafeína/mL).

Com relação aos resultados do colesterol ligado à lipoproteína de muito baixa densidade (VLDL colesterol) (Figura 4), observou-se uma diminuição altamente significativa $(\mathrm{p}<0,01)$ desta nos ratos pertencentes ao grupo 3 quando comparados aos do grupo controle e aos dos demais grupos em que se administrou cafeína em concentrações mais baixas.

Ficou caracterizado nesses resultados um efeito dose-dependente da cafeína sobre os níveis séricos de colesterol total, triglicerídeos, HDL colesterol e VLDL colesterol. 


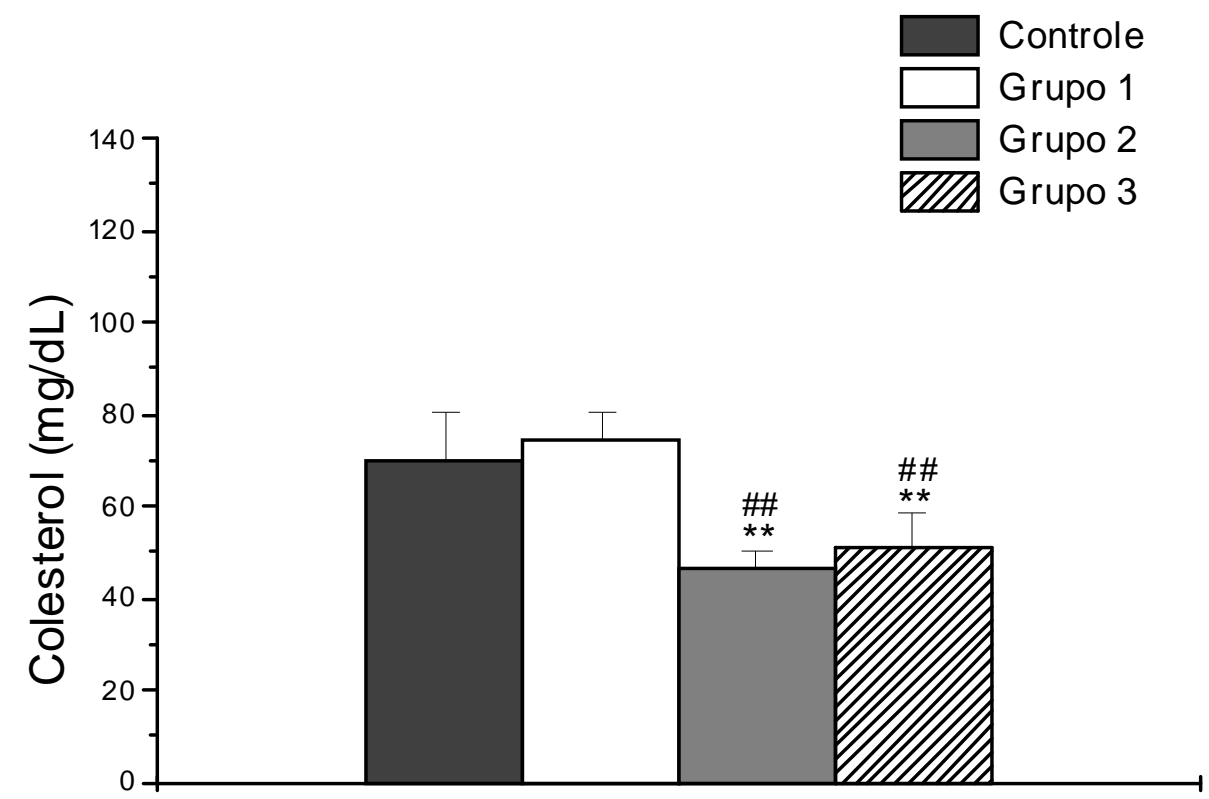

Figura 1 - Colesterol Total na ausência (grupo controle) e presença de cafeína em diferentes concentrações: $0,3 \mathrm{mg} / \mathrm{mL}$ (grupo 1); $6 \mathrm{mg} / \mathrm{mL}$ (grupo 2) e $10 \mathrm{mg} / \mathrm{mL}$ (grupo 3).

Fonte: Dados de pesquisa.

$* * \mathrm{p}<0,01$ : significativamente diferente em relação ao grupo controle.

$\# \#$ p < 0,01: significativamente diferente em relação ao grupo 1.

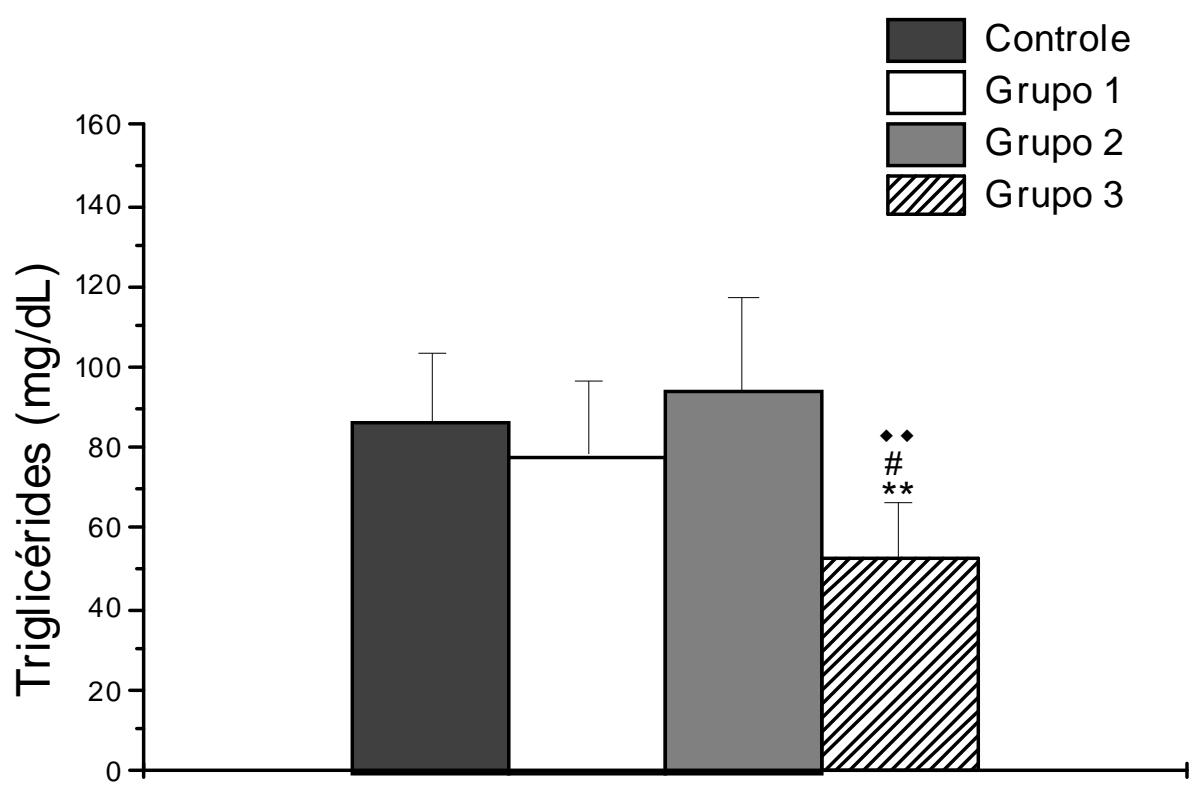

Figura 2 - Triglicérides na ausência (grupo controle) e presença de cafeína em diferentes concentrações: 0,3 $\mathrm{mg} / \mathrm{mL}$ (grupo 1); $6 \mathrm{mg} / \mathrm{mL}$ (grupo 2) e $10 \mathrm{mg} / \mathrm{mL}$ (grupo 3).

Fonte: Dados de pesquisa.

$* * \mathrm{p}<0,01$ : significativamente diferente em relação ao grupo controle.

\# p< 0,05: significativamente diferente em relação ao grupo 1.

$\bullet$ : $\mathrm{p}<$ 0,01: significativamente diferente em relação ao grupo 2. 


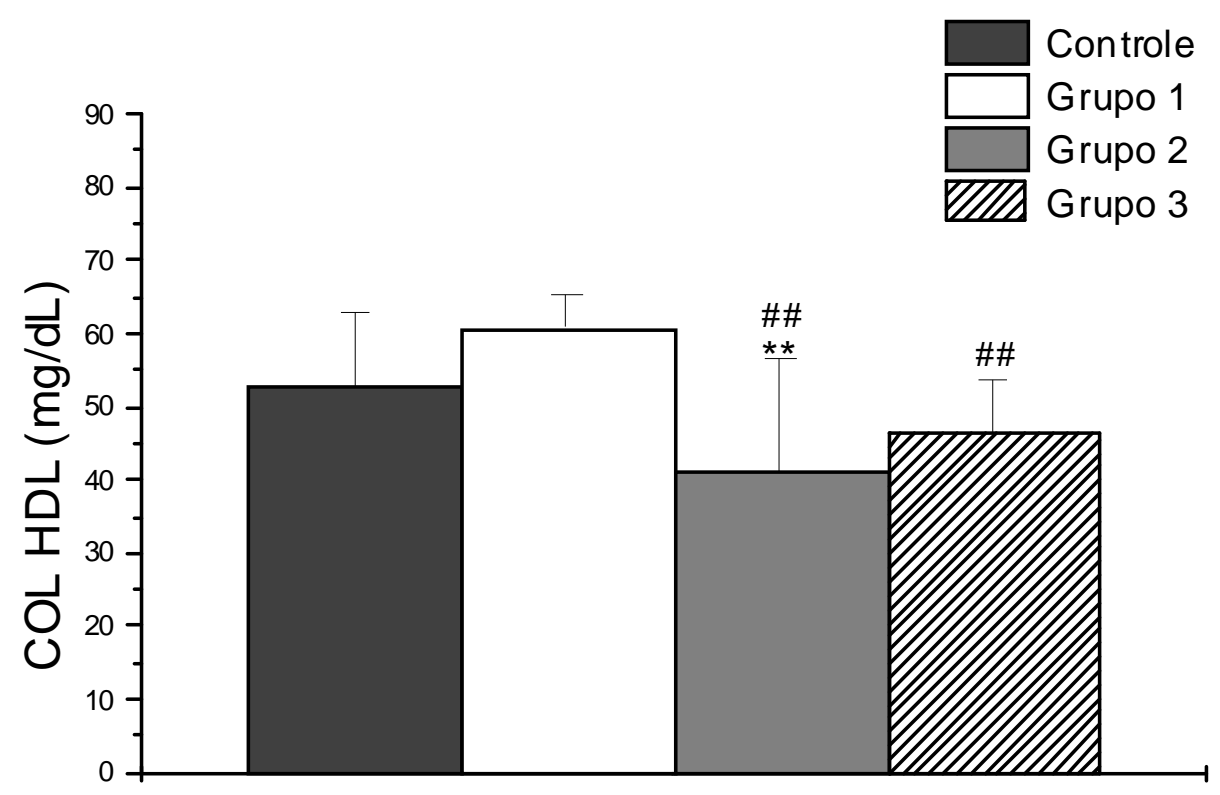

Figura 3 - HDL colesterol na ausência (grupo controle) e presença de cafeína em diferentes concentrações: $0,3 \mathrm{mg} / \mathrm{mL}$ (grupo 1 ) $; 6 \mathrm{mg} / \mathrm{mL}$ (grupo 2) e $10 \mathrm{mg} / \mathrm{mL}$ (grupo 3).

Fonte: Dados de pesquisa.

** $\mathrm{p}<0,01$ : significativamente diferente em relação ao grupo controle.

$\# \#$ p < 0,01: significativamente diferente em relação ao grupo 1.

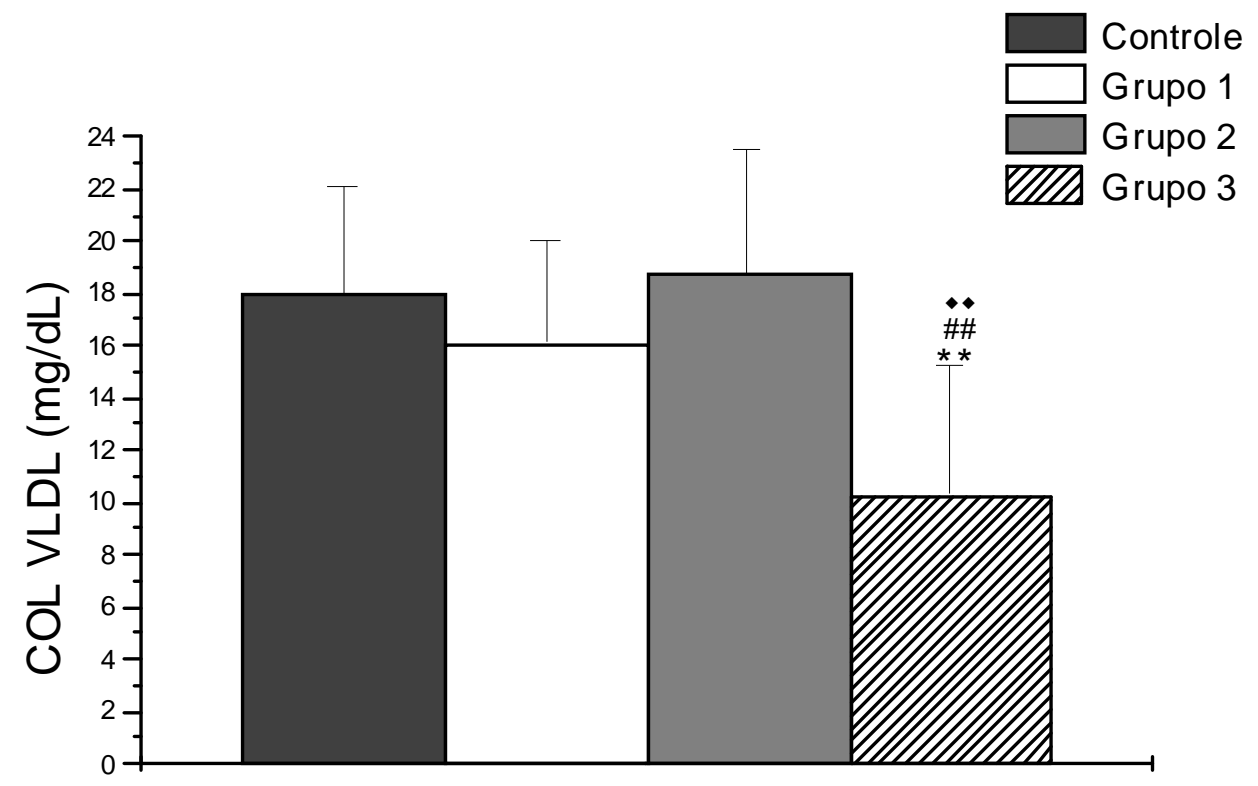

Figura 4 - VLDL colesterol na ausência (grupo controle) e presença de cafeína em diferentes concentrações: $0,3 \mathrm{mg} / \mathrm{mL}$ (grupo 1); $6 \mathrm{mg} / \mathrm{mL}$ (grupo 2) e $10 \mathrm{mg} / \mathrm{mL}$ (grupo 3).

Fonte: Dados de pesquisa.

** $\mathrm{p}<0,01$ : significativamente diferente em relação ao grupo controle.

$\# \# \mathrm{p}<0,01$ : significativamente diferente em relação ao grupo 1.

$\bullet \bullet$ p $<0,01$ : significativamente diferente em relação ao grupo 2 . 


\section{Discussão}

O café é uma das bebidas mais ingeridas em todo o mundo. Devido ao seu alto consumo em maior parte dos países, é interessante, tanto do ponto de vista público quanto de uma perspectiva científica, a discussão de seus benefícios potenciais ou seus aspectos negativos em relação aos dois principais problemas de saúde, ou seja, doenças cardiovasculares e diabetes mellitus tipo 2 (RAHEIM; HALVORSEN, 2005).

O presente trabalho demonstrou uma diminuição significativa nos níveis de colesterol total, triglicerídeos, VLDL colesterol e HDL colesterol principalmente em altas doses de cafeína, corroborando resultados de trabalhos anteriores (HACKMAN et al., 2006; COSTA, 2004).

Esses resultados demonstram que o consumo de cafeína poderia ajudar a reduzir o risco de doença coronariana e ser útil como instrumento na perda de peso, devido a sua ação sobre os lipídeos (colesterol total, triglicerídeos e VLDL colesterol); o maior problema observado neste trabalho foi a redução nos níveis séricos do HDL colesterol, que reduziria, assim, o efeito cardioprotetor.

Estudos epidemiológicos demonstram que o consumo de café fervido e não filtrado eleva o risco de doenças cardiovasculares, em razão da presença de dois diterpenos na fração lipídica do café - o cafestol e kahweol -, que promovem o aumento da concentração plasmática de colesterol em seres humanos. Contradizendo esses estudos, alguns trabalhos demonstraram uma diminuição na concentração plasmática de colesterol na presença de cafeína e assinalam, como possível mecanismo, uma diminuição dos limites de absorção do colesterol pelo intestino, ocasionada pelo consumo de chá preto de forma mais significativa, quando comparado ao consumo apenas de cafeína (DAVIES et al., 2003). Por outro lado, o café é uma fonte rica em compostos heterocíclicos, que apresentam forte atividade antioxidante, sugerindo um efeito benéfico do mesmo no sistema cardiovascular (CORNELIS; ELSOHEMY, 2007). Além disso, algumas referências na literatura sugerem que a cafeína aumente a liberação de lipase, que tem ação sobre os lipídeos (IFIC, 1999).

Vários trabalhos apontam que a investigação epidemiológica do consumo de café pode prevenir diversas doenças crônicas, como diabetes mellitus tipo 2, doença de Parkinson e doenças hepáticas (cirrose e carcinoma hepatocelular) (HIGDON, 2006).

Estudos apontam que consumidores regulares de cafeína ficam irritadiços, ansiosos ou com dor de cabeça se não consumirem sua dose diária da mesma (BURGOS, 2008). Outros estudos demonstram que, quando o consumo ultrapassa $200 \mathrm{mg}$ diárias, independente da fonte, a cafeína perde seu efeito analgésico e pode causar dor de cabeça (BUCHALLA, 2008).

Hoje, além de consumir a cafeína no café, chás, chocolates, refrigerantes e analgésicos, as pessoas a tem usado como "droga utilitária" para se manter acordadas. Como consequência, as pessoas dormem menos e aumentaram muito os problemas vinculados à falta de sono, como acidentes de trânsito e até mesmo impotência sexual (BURGOS, 2008).

As doses de cafeína em que se obtiveram resultados significantes foram muito altas, e seus benefícios precisam ser avaliados com muito critério. A ingestão de cafeína deve ser revista com prudência, principalmente por pessoas mais suscetíveis aos seus efeitos adversos, como gestantes, crianças, hipertensos, entre outros.

\section{Conclusão}

Diante do seu efeito sobre o perfil lipídico, a cafeína pode ser também um coadjuvante interessante na prevenção de doenças cardiovasculares e perda de peso, devido à grande quantidade de alimentos e demais substâncias ricas em CF que são ingeridas diariamente pela população em geral, pois atua diminuindo os níveis séricos de colesterol total, triglicerídeos e VLDL colesterol. Porém, deve-se considerar que também houve uma redução nos níveis séricos de HDL colesterol, evidenciando, portanto, a redução da lipoproteína HDL, uma molécula 
cardioprotetora, fazendo-se necessário reavaliar os seus efeitos sobre o sistema cardiovascular.

\section{REFERÊNCIAS}

BRASILESCOLA. Disponívelem: www.brasilescola.com/drogas/cafeina.htm, acesso em 05/abr./2007.

BUCHALlA, A. P. Brasil, País da Dor de Cabeça. Veja. v. 21. ed. 2062, p. 88-95, Maio de 2008.

BURGOS, P. À Base de Cafeína. Super

Interessante. n. 254, p.76-81, Jul. de 2008.

CAFÉ E SAÚDE. Disponível em:

www.cafeesaude.com.br, acesso em 15/set./2007.

CAFEÍNAADROGAPREDILETA. Disponível em: www.qmc.ufsc.br/qmeweb/artigos/cafeína.html, acesso em 15/set./2007.

CAMARGO. Disponível em: www.br.geocitie.com/ raosanacamargo2001/cafeina naalimentação.html, acesso em 08/maio/2007.

CLOSE, B. et al. Recommendations for euthanasia of experimental animals: Part 1. Laboratory

Animals. v. 30. p. 293 - 316, 1996.

COLESTEROL. Disponível em:

www.fisiologia.kit.net/bioquimica/lipidios/image2.jpg, acesso 15/jul./2007

CORNELIS, M. C.; EL - SOHEMY, A. Coffee, Caffeine, and Coronary Heart Disease. Curr Opin Lipidol. 2007.

CURI et al. Entendendo a Gordura: Os Ácidos Graxos. Barueri: Manole, 2002.

DAVIES et al. Black tea consumption reduces total and LDL cholesterol in mildly hypercholesterolemic adults. American Society for Nutritional

Sciences, 2003.

FREDHOLM, B. B. Adenosine, Adenosine Receptors and Actions of Caffeine. Pharmacology \& Toxicology. v. 76, p. 93-101, 1995

GEORGE, S. E.; RAMALAKSH, K.; MOHAN RAO, L. J. A Perception on Health of Coffee. Central Food Technological Research Institute. p. 464 - 486, 2008.

HACKMAN et al. Multinutrient supplement containing ephedra and caffeine causes weight loss and improves metabolic risk factors in obese women: a randomized controlled trial. International Journal Obesity, v.30, p. 1545-1556, 2006

HIGDON, J. V.; FREI, B. Coffee and health: a review of recent human research. Critical Reviews in Food Science and Nutrition, v.46, n.2, p. 101123, 2006.

HOLTZMAN, S.G.; MANTE S.; MINNEMAN, K. P. Role of Adenosine Receptors in Caffeine Tolerance. Journal of Pharmacology and Experimental Therapeutics. v. 256, 1991.

INTERNATIONAL FOOD INFORMATION COUNCIL FOUNDATION (IFIC). Caffeine \& Health: Clarifying the controversies, 1999.

JAMES, J. E. Understanding Caffeine-a biobehavioral analysis. London: Sage, 1997.

KAPLAN, G. B.; TAI, N. T.; GREENBLATT, D.J. \& SHADER, R.I. Separate and Combinede Effects of Caffeine and Alprazolam on Motor Ativite and Benzodiacepine Receptor Binding in Vivo.

Psychopharmacology. v. 101. p. 539-544, 1990

NEWSLETTER. Disponível em: 
www.newsletter.minhavida.com.br, acesso em 08/ maio/2007.

MICHAELIS, M. L.; MICHAELIS, E. K. \& MYERS, S. Adenosine Modulation of Senaptosomal Dopamine Release. Life Sciences. v. 24. p. 2083-2092, 1979.

MIKKELSEN, E. J. Caffeine and Schizophrenia. Journal of Clinical Psychiatry. v. 39. p. 732-736, 1978.

POLBR. Disponívelem: www.polbr.med.br/arquivo/ mess0801.htm, acesso em 09/abr./2007.

PSIQWEB. Disponível em: www.psiqweb.med.br/ farmaco/cafeina.html, acesso em 08/jun./2007.

PSIQWEB. Disponível em: www.psiqweb.med.br/ farmaco/cafeina.html, acesso em 18/maio/2007.

RANHEIM, T; HALVORSEN, B. Coffee consumption and human health-beneficial or detrimental? Mechanisms for effects of coffee consumption on different risk factors for cardiovascular disease and type 2 diabetes mellitus. Mol Nutr Food Res, v.49, n.3 p. 274284, 2005.

SNYDER, S. H. Adenosine as a Midiator of the Behavioral Effects of Xanthines. In: Dews, P. B. Caffeine: perspectives from recent research. Berlin: Springer-Velag. 1984. p. 129-141.

SOARES; FONSECA. Disponível em: www.ff.up.pt/toxicologia/monografiasano0405/ cafeína/cafeína.pdf, acesso em 27/maio/2007.

STRAIN, E. C.; GRIFFITHS, R. R. Caffeine Related Disorders. In: SADOCK, B. J.; SADOCK, V.A. Kaplan \& Sadock's Comprehensive Textbook of Psychiatry - 7.ed. Baltimore: Lippincott, 2000. p. 982-990.

WALDECK, B. Some Effects of Caffeine Aminophylline on the Turnover of Catecholamines in the Brain. Journal of Pharmacy and Pharmacology. v. 23.p. 824-830, 1971.

RECEBIDO EM 15/12/2010

ACEITO EM 29/6/2011 